\title{
Enzyme Therapy that Can Digest the Toxic Motifs of Gluten as an Aid in the Management of Celiac Disease
}

\author{
Finlay Macrae AO* \\ Department of Medicine, University of Melbourne, The Royal Melbourne Hospital, Australia \\ *Corresponding author: Finlay.Macrae@mh.org.au
}

\begin{abstract}
For the first time since discovery of celiac disease and introduction of gluten-free diet (GFD) there are some treatments that could be called adjuncts to the GFD for the management of celiac disease (CD) and dermatitis herpetiformis $(\mathrm{DH})$. The most clinically advanced approaches are based on enzymatic detoxification of traces of gluten often present in seemingly gluten-free foods. Maintenance of GDF is difficult for many patients due to undeclared levels of gluten even in "gluten free" products. Enzyme supplementation enables detoxification of "hidden" gluten and guards against gluten before it can damage intestinal mucosa and stimulate the immune system. Controversy rests as to whether such advances should be encouraged for patients with celiac disease, whose behaviour then might impact on their commitment to maintain a gluten free diet.
\end{abstract}

Keywords: celiac disease, enzyme therapy, gliadin peptides, dermatitis herpetiformis, caricain, gluten, clinical studies

Cite This Article: Finlay Macrae AO, "Enzyme Therapy that Can Digest the Toxic Motifs of Gluten as an Aid in the Management of Celiac Disease." International Journal of Celiac Disease, vol. 6, no. 1 (2018): 4-6. doi: 10.12691/ijcd-6-1-3.

\section{Introduction}

About half of Australian coeliac patients fail to heal their bowel or suffer persistent symptoms even after many years on a gluten free diet. The cause of ongoing symptoms in people with celiac disease (CD) is often attributed to accidental or low amounts of gluten exposure leading to persistent intestinal inflammation. Currently the Coeliac Society of Australia is funding a large study at the Walter and Elisa Hall Institute in Melbourne titled "How gluten free is a gluten free diet?" with preliminary results indicating substantial levels of gluten contaminating many gluten free products [1].

CD affects an estimated $1 \%$ of Caucasian people globally and is triggered by glutens in wheat, rye, barley and some varieties of oats [2]. It is one of the most common food-related lifelong disorders in the world [3].

$\mathrm{CD}$ is normally detected by serological tests and confirmed by duodenal biopsy when the patient is on a normal gluten-containing diet. Symptoms usually found are abdominal pain, bloating, diarrhea as well as fatigue and other symptoms related to malnourishment due to damaged small intestine. The prevalence of diagnosed $\mathrm{CD}$ has increased fourfold to fivefold over the past 50 years. Despite advances in our understanding of its pathophysiology and improved diagnostic tools, the rise in $\mathrm{CD}$ has been a largely silent epidemic, with undiagnosed patients going untreated. Persistently active mucosal disease in celiac patients is associated with an increased risk of lymphomas, some cancers [3] and mortality [4].
CD results from the interaction of environmental factors, mainly gluten, with immune and genetic factors. The disease occurs almost exclusively in people with the DQ2 or DQ8 HLA haplotype. Celiac patients with these HLA haplotypes are unable to break down gluten to smaller peptides which do not induce immunological responses or direct epithelial damage $[5,6]$.

Clearly, to develop abnormal immune responses to gluten, the toxic and immunogenic peptides of gluten would have to be present in the gut and to which the immune system needs to react. However, if enzymes in the gut digest these peptides into harmless smaller fragments, development of such an immune response, and the associated symptoms, would be averted. Enzyme deficiency in $\mathrm{CD}$ was formally demonstrated in the early 1970s when the duodenal mucosa of children with CD was found to lack an enzyme [7] which was later confirmed by organ culture [8]. The deficient enzyme was determined to be an endopeptidase, which, if present, would digest the toxic gluten peptides into harmless fragments. This finding is supported by a bread baking study where addition of endopeptidase (caricain) to the dough reduced toxicity of gluten by more than $90 \%$ [9]. Caricain is the active ingredient of an enzyme preparation recently developed and now available in Australia as aid for the digestion of gluten. .

Currently, the only known treatment for $\mathrm{CD}$ is adherence to a lifelong strict gluten-free diet. Although a gluten free diet is effective when implemented correctly, many patients find it difficult to follow. It is expensive, with bread and pasta substitutes costing substantially more than their gluten-containing counterparts. It can be 
socially isolating in settings such as communal celebrations, religious rituals, and dining out. Strict avoidance of gluten can be a big challenge because of hidden sources of gluten. Primary sources of gluten are wheat, rye, barley and some varieties of oats [2]. Common hidden sources of gluten are sauces, drugs (prescription and over the counter items, including dietary supplements), shared food preparation and processed meats. Potential hidden gluten in drugs or sauces is a legitimate source of anxiety for patients, including those who have acute symptoms on minimal exposure to gluten.

The enzyme therapy concept was developed in Melbourne by Prof Hugh Cornell and Dr Ted Stelmasiak [10]. The world first clinical trial of an enzyme supplement for celiac disease was conducted at The Royal Melbourne Hospital in early 2005. This study was based upon oral administration of an animal derived endopeptidase. Amelioration of symptoms occurred and signs of protection as assessed histologically were seen even when volunteers were challenged with $1 \mathrm{~g}$ of gluten daily [7]. Due to emergence of animal viruses and prions capable of infecting humans, the animal derived enzymes were abandoned in favour of other alternatives. The next generation product is based on a papaya fruit enzyme called caricain (GluteGuard, Glutagen, Melbourne, Australia).

A number of approaches in non-dietary treatments have been investigated around the world, including the enzymatic detoxification of ingested gluten, a vaccine to induce gluten tolerance, inhibitors of the HLA DQ2 and DQ8 proteins or tissue transglutaminase antibodies (tTG) and others [12].

The enzyme supplements are the most advanced treatments available. To date, two enzyme treatments, an American and the Australian one, have been evaluated in clinical studies involving gluten challenge. In Finland, the American product called ALV003, based on recombinant enzymes, was compared with a placebo in patients with CD. Sixteen patients were given the enzyme and 18 given placebo. Those receiving enzyme had no significant reduction in villous height on intestinal biopsy, whereas those taking placebo had the expected gluten induced mucosal injury [13]. ALV003 appeared to attenuate gluten-induced small intestinal mucosal injury in patients with $\mathrm{CD}$ in the context of an everyday gluten-free diet containing up to $2 \mathrm{~g}$ gluten daily.

The Australian product called GluteGuard was evaluated in two collaborative clinical studies in Poland involving celiac and dermatitis herpetiformis patients.

\section{CD Clinical Trial}

The Polish celiac study [14] was a randomised double blind trial of $20 \mathrm{CD}$ patients in clinical remission on a gluten-free diet. All patients ingested 1 gram of gluten (equivalent to approximately half a slice of bread) each day for 42 days, with 14 of them taking Glute Guard at the same time and 6 taking a placebo tablet. Patients recorded their CD symptoms and wellbeing each day, and intestinal tissue was examined before and after the study.

Thirteen of 14 CD patients taking Glute Guard (93\%) demonstrated no detrimental changes in clinical symptoms, biopsy results or wellbeing throughout 42 days of gluten challenge. Conversely, 4 of $6(67 \%)$ taking placebo developed severe CD symptoms and withdrew from further gluten challenge after 14 days, compared to 1 of 14 $(7 \%)$ from the treatment group $(p<0.001)$. Comparing the therapy and placebo groups across the first 14 days of gluten challenge, those taking Glute Guard reported fewer CD symptoms (28 vs 118 symptom points, respectively; $p$ $<0.01$ ) and $38 \%$ higher wellbeing scores (8.4 vs 6.1, respectively; $\mathrm{p}<0.01)$. There was attenuation of mucosal injury in the treatment group, similar to the Finnish study. Although these analyses were not according to intention to treat, the signal for protection was strong in the data. If patients on placebo who dropped out (mostly because they developed symptoms relating to gluten exposure) were included, such an intention to treat analysis would be even stronger. Furthermore, the results verified the in vitro work on toxic epitopes of gluten referred to earlier in the papers $[5,6]$ and lead to better understanding of enzyme therapy.

\section{DH Clinical Trial}

The other Polish study investigated the effectiveness of GluteGuard in patients with dermatitis herpetiformis (DH) [15], a skin condition similar to CD in that it is triggered by intolerance to gluten peptides. Like $\mathrm{CD}$, a strict lifelong gluten-free diet is essential for managing $\mathrm{DH}$ and preventing skin inflammation and other complications.

In this second Polish randomised double blind study, this time of DH patients largely in clinical remission, all patients consumed approximately 6 grams of gluten daily for 7 days, with 10 concurrently taking GluteGuard tables and 10 placebo. GluteGuard offered a significant $81 \%$ protection by reducing the area of skin lesions from 19.5 $\mathrm{cm} 2$ to $3.7 \mathrm{~cm}^{2}$, (placebo vs caricain treatment, respectively; $\mathrm{p}=0.02$ ), a substantial $71 \%$ reduction in the appearance of skin lesions (24 lesions vs 7, respectively), and a 38\% reduction in emergence of troublesome itch (40 vs 25 episodes, respectively). Of $7 \mathrm{DH}$ patients who withdrew from the study due to gluten challenge-related symptoms, 6 were taking placebo. For such a modest trial, the results were remarkable.

The number of subjects in each of these trials was small; even so, clinical and histological benefit was demonstrable pointing to the potency of the approach of enzyme supplementation

\section{Conclusions}

Caricain-derived enzyme supplementation promises to be a useful adjunct for $\mathrm{CD}$ and $\mathrm{DH}$ patients anticipating unavoidable exposure to gluten (e.g. travelling, cuisine prepared outside their direct control) or for those particularly sensitive to gluten. The trials were some of the first to demonstrate efficacy of enzyme therapy to support people with CD. The enzyme strategy seems logical since it can detoxify gluten before it induces any intestinal damage and before stimulation of the immune 
system occurs. Caricain is harmless itself and specifically targets gluten. This enzyme therapy will not cure CD or $\mathrm{DH}$ but it can help in digesting hidden dietary gluten, which on the existing trial evidence, limits mucosal damage and makes life easier for many patients with coeliac or DH.

Notwithstanding the evidence outlined above, some specialists have concern that patients with coeliac disease will relax their gluten free diets sufficiently liberally to overwhelm the protection that enzyme therapy can afford. This is akin to not offering antihypertensive therapy to people whose blood pressure increases in response to salt in their diets, lest they relax that dietary advice. It second guesses the behaviour of celiac patients, including all those symptomatic patients who would benefit despite adhering faithfully to a "gluten free" diet. A valid concern however, is the lack information, as yet, on the long term benefits (or harms) of enzyme therapy in patients with celiac disease.

To be sure, patients with coeliac disease should not deviate from their gluten free diet. But enzyme supplementation can, however, assist in their often difficult task of managing their disease. Confidence in the value of enzyme therapy has been boosted by the results of clinical trials of patients with two diseases in which gluten is central to their pathogenesis. Indeed, caricain-derived endopeptidase has been singled out for commendation by Professor Aaron Lerner: "Gluteguard was able to protect patients from adverse symptoms being induced by gluten challenge. The advantages of the fruit originated preparation are: it does not contain living microbes or bacterial purified engineered products. It can be considered as preventive therapy and a further step in the future therapeutic strategy race for the celiac population benefits" [16].

Acknowledgement: The author would like to warmly acknowledge the lifelong scientific career of Prof Hugh Cornell who has built the evidence around deficiency endopeptidases in the pathogenesis of coeliac disease

Statement of Competing Interests: Glutagen Pty Ltd, manufacturer of Gluteguard, provided funding assistance to support the early trials of the pig derived endopeptidase conducted by the author, and some funding assistance for the trials done in Poland.

\section{References}

[1] Halmos E. How gluten free is gluten free diet. (2016) The Australian Coeliac, December Issue. p.11-1.

[2] Silano M, Dessi M, De Vincenzi M, Cornell $H$. In vitro tests indicate that certain varieties of oats may be harmful to patients with celiac disease. (2007) J GastroenterolHepatol 22; 528-531.

[3] Lebwohl B.,Ludvigsson J.F, Green P.H.R. Celiac disease and nonceliac gluten sensitivity. (2016) Published online PMCID: PMC4596973.

[4] Ludvigsson JF, Montgomery SM, Ekbom A, et al. Small-intestinal histopathology and mortality risk in celiac disease.(2009) JAMA302: 1171-8.

[5] Cornell H. J., Stelmasiak T. The Significance of Key Amino Acid Sequences in the Digestibility and Toxicity of Gliadin Peptides in Celiac Disease. (2016) International Journal of Celiac Disease 4: 113-120.

[6] Cornell, HJ. Partial in vitro digestion of active gliadin-related peptides in coeliac disease. (1998) J Protein Chem 17; 739-44.

[7] Cornell, HJ, and Townley, RR. Investigation of possible intestinal peptidase deficiency in coeliac disease. (1973) Clinica Chimica Acta 43: 113-125.

[8] Townley, RRW, Cornell, HJ, Bhathal, PS, and Mitchell, JD Toxicity of wheat gliadin fractions in coeliac disease. (1973) Lancet 1(7816): 1363-4.

[9] Buddrick O, Cornell HJ, Small DM. Reduction of toxic gliadin content of wholegrain bread by the enzyme caricain. (2015) Food Chem. 170:343-7.

[10] Cornell, HJ, and Stelmasiak, T. A unified hypothesis of coeliac disease with implications for management of patients. (2015) Amino Acids 233: 43-9.

[11] Cornell H J, Macrae F A, Melny J, Pizzey C, Cook F, Mason S, Bhathal P \&Stelmasiak T. Enzyme therapy for management of coeliac disease. (2005) Scand. J. Gastroent. 40: 1304-1312.

[12] Wungiranirun M, Kelly C.P. and Leffler D A. Current status of celiac disease drug development. (2016) J Gastroenterology.

[13] Lahdeaho ML, Kaukinen K, Laurila K, et al. Glutenase ALV003 attenuates gluten-induced mucosal injury in patients with celiac disease. (2014) Gastroenterology 146: 1649-58.

[14] Cornell H J,Czyzewska A , Macrae F A, Rydzewska G, Nasierowska-Gutmejer A, Bednarczuk A and Stelmasiak T. The Effect of Enzyme Supplementation on Symptoms and Duodenal Histology in Celiac Patients.(2016) International Journal of Celiac Disease. 4: 40-47.

[15] Zebrowska A, Cornell HJ, Macrae FA, Sysa-Jedrzejowska A, Waszczykowska A, Stelmasiak T. The effect of enzyme therapy on skin symptoms and immune responses in patients with dermatitis herpetiformis. (2014) International Journal of Celiac Disease. 2: 58-63.

[16] Lerner A. Is enzyme supplementation effective strategy to reduce the burden of gluten free diet in celiac disease? (2016) International Journal of Celiac Disease 4:38-39. 